\title{
Dynamics of growth and signaling along linear and surface structures in very early tumors
}

\author{
ANNA MARCINIAK-CZOCHRA $\dagger^{*}$ and MAREK KIMMEL $\$$ § \\ $\dagger$ Institute of Applied Mathematics, Center for Modeling and Simulations in the Biosciences (BIOMS), University of \\ Heidelberg, Im Neuenheimer Feld 294, 69120, Heidelberg, Germany \\ \$Department of Statistics, Rice University, 6100 Main St, MS-138, Houston, TX 77005, USA \\ I Systems Engineering Group, Silesian University of Technology, Akademicka 16, 44-100, Gliwice, Poland
}

(Received 1 June 2006)

\begin{abstract}
There exists evidence that in early stages tumors progress along linear, tubular, or irregular surface structures. This seems to be the case for atypical adenomatous hyperplasia (AAH), a precursor of adenocarcinoma of the lung. We previously published a simplified model, which showed that early structures had a potential towards spontaneous invasive growth following a latency phase. The transition was facilitated by diffusion of a growth factor and nonlinear cell cycle regulation in cancer cells. The mechanism is analogous to that in Turing pattern formation, although the patterns are irregular and unstable. We introduce more biologically justifiable signaling, in which only the free growth factor molecules diffuse. Flexible nonlinearities in the model accommodate several growth patterns of cells as well as internal versus external production of the growth factor. We show that the reaction-diffusion setup results in complicated spike-like solutions. We discuss these results in the light of published data on the AAH.
\end{abstract}

Keywords: Cancer modelling; Atypical adenomatous hyperplasia (AAH); Bronchoalveolar carcinoma (BAC); Reaction-diffusion equations; Pattern formation; Spike solutions

\section{Introduction}

The earliest stages of cancer development are still a great unknown. The most common view involves the initial single malignant cell, which then produces a clone of genetically identical cells, which first grow as a primary tumor and continuously evolve, and then the most malignant of them, following a series of genetic changes, settle in lymph nodes and distant organs and form metastasis. This is a simplified view, which does not take into account the details of early invasion nor the importance of communication between malignant cells. Recently, new experimental evidence was obtained concerning early spatial organisation of various tumors, including lung cancers. Also, for the first time, it became possible to obtain radiographic images (CT scans) of tumors in patient's lungs, which can be directly compared to pathological specimens from surgically excised tumors (figure 1). In this paper, we will follow up on our previous work and try to explain the pattern of early invasion of precursors

*Corresponding author. Tel.: +49-622-154-8971. Fax.: +49-622-154-5331. Email: anna.marciniak@iwr.uniheidelberg.de

${ }^{\S}$ Tel.: + 17-133-485-255. Fax.: +17-133-485-476. Email: kimmel@rice.edu. 


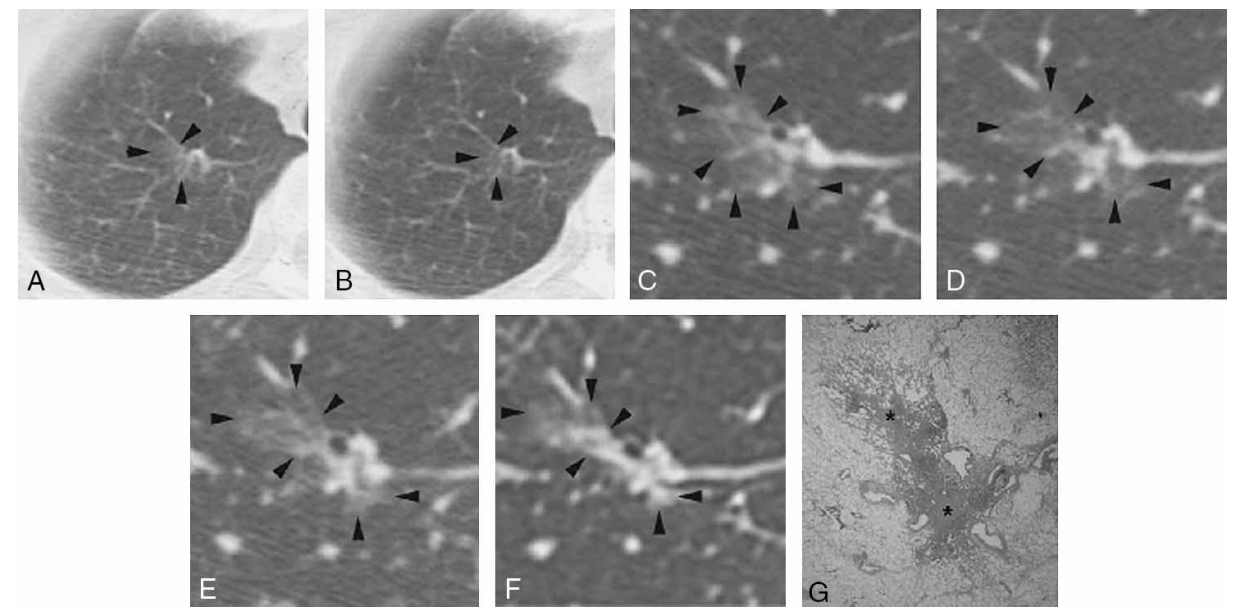

Figure 1. Bronchioloalveolar carcinoma in a 69-year-old man. (A) A faint localised increase in density was identified in segment 1 of the right upper lobe of the lung on a CT screening image obtained in February 1999. (B) In retrospect, the opacity was also visible on a CT screening image obtained in February 1998. (C) Thin-section CT revealed a pGGO in segment 1 of the right upper lobe of the lung in March 1999. (D) Thin-section CT image obtained in February 2000 showing a pGGO with a small solid component. (E) Thin-section CT image obtained in February 2001 showing a decrease in the size of the pGGO and a slight increase in the size of the solid component. (F) Thin-section CT image obtained in February 2002 showing a larger decrease in the size of the pGGO and an increase in the size of the solid component. (G) Low-magnification image of the pathologic specimen. The foci of alveolar collapse (asterisks) are shown. A right upper lobectomy was performed in May 2002. The lesion was diagnosed as a bronchioloalveolar carcinoma, $15 \mathrm{~mm}$ in diameter (Noguchi type B) (reproduced from [12] by permission from Lippincott, Williams and Wilkins).

peripheral lung tumors. We will use models, which attempt in simplified geometry to reproduce the irregular spikes of invasion in the early lesions (figure 2, panels $\mathrm{C}$ and $\mathrm{D}$ ).

\subsection{Early peripheral lung tumors}

The best-known theory of carcinogenesis has been developed by Moolgavkar [19]. It is known as the Two-Stage Clonal Proliferation Model. According to Moolgavkar's theory, normal cells require two transformations, before they form tumors: Stage 1, called Initiation, confers the ability to proliferate. In Stage 2, one of the proliferating initiated cells undergoes a Promotion event, which makes it malignant. Among many specific consequences of this model, there is one, which stands out: population of cells constituting the tumor is clonal, i.e. it is part of the progeny of the single promoted cell. It is an interesting question whether this theory stands the test of more recent observations. The answer might be provided by observation and analysis of very early cancerous or pre-cancerous lesions and their patterns of progression.

In lung cancer, there has been a constant progress in visualisation of early stages of the disease, for example using computed tomography [12,23], and correlating these findings with structures observed in biopsy or surgery specimens. Among the most interesting recent findings are those of the apparent progression from the atypical adenomatous hyperplasia (AAH) to bronchoalveolar carcinoma (BAC) to adenocarcinoma of the lung, [13]. Adenocarcinoma is one of the major subtypes of lung cancer, which is usually located peripherally with respect to the bronchial tree in the lung. The relative incidence of adenocarcinoma compared to other lung cancer cell types seems to be increasing, due to an 


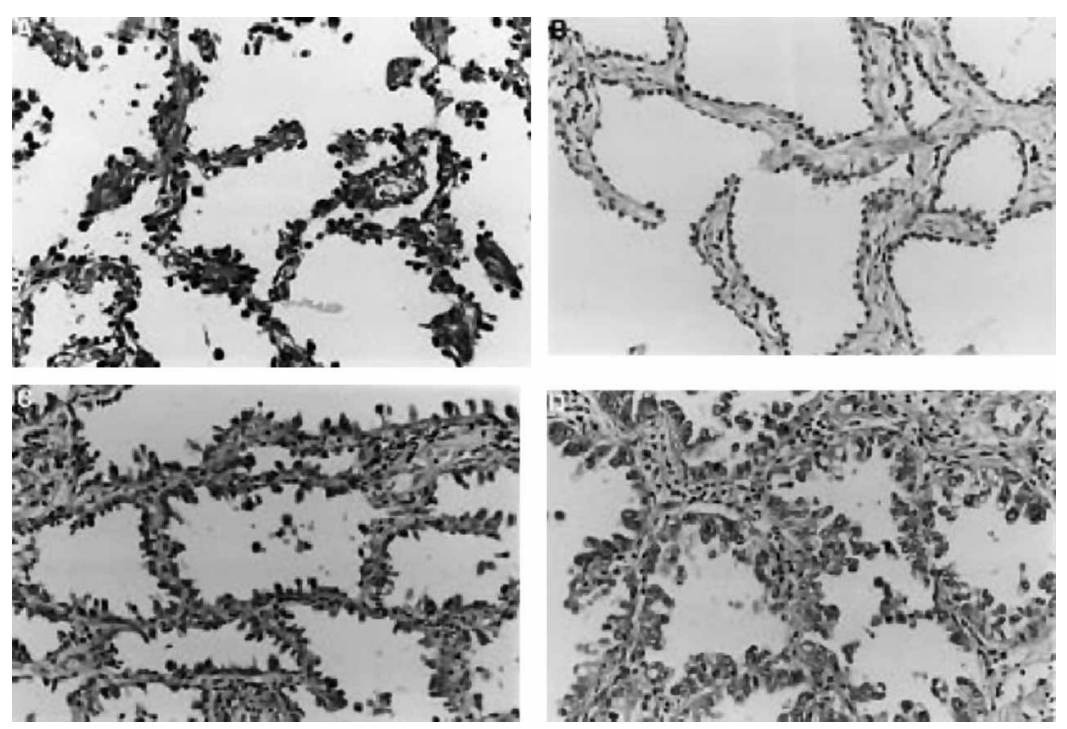

Figure 2. AAH. Low grade lesions show (A) intermittent or (B) complete runs of cuboidal cells lining slightly thickened alveolar walls. More cellular lesions (C) may be larger and have columnar Clara-like cells. Less often, lesions are very cellular, more atypical (D), and very difficult to distinguish from bronchioloalveolar cell carcinoma (reproduced from [13], by permission from BMJ Journals).

increase in consumption of filter cigarettes [6]. A 20-year old study of unresected Stage I socalled BAC showed progression of all 48 tumors in 2 years and a frequently fatal outcome in 3 years, about $10 \%$ of cases progressing more slowly but with the same eventual outcome [11]

It has been observed that incidence of AAH as a histological entity can be correlated with the so-called ground-glass opacities observed in CT images [12,23]. These lesions have the form of diffuse systems of thin lines and shades, sometimes with solid nodules inside, and are frequently observed to grow and progress to adenocarcinoma during the period of months or years (see figure 1 reproduced from [12]). On the other hand, AAH seems to initially have the form of branches of tubular sheets of cells lining the surfaces of the fine branchings of the bronchial tree [13]. Subsequently, these cells seem to invade the adjacent tissue matrix. The analogy with the natural history of the GGOs seems justified. Figure 2 reproduced from [13] presents histological specimens of AAH. Low-grade lesions show (A) intermittent or (B) complete runs of cuboidal cells lining slightly thickened alveolar walls. More cellular lesions (C) may be larger and have columnar Clara-like cells. Less often, lesions are very cellular, more atypical (D), and very difficult to distinguish from BAC. The objective of our model is to elucidate the possible mechanism of transition from structures of type (A) or (B) to structures (C) and (D). We propose that the causal mechanism may be diffusion of growth factor particles over the cell sheets forming the AAH combined with cell cycle regulation.

What is interesting about AAH lesions is that they first take the highly structured form and then, apparently after a time delay, penetrate in an irregular way the adjacent tissue. Also, they seem to be clonal, in the sense of identical genetic makeup [21]. As a response to cell crowding, they seem to increase expression of metalloproteases, which allow them to penetrate the tissue matrix. Therefore, although the AAHs could be candidates for the initiated cells of the Moolgavkar's model, the question as to whether the BACs are the promoted cells is quite open. In particular, the local progression of BACs seems to take place 
in a contiguous stretch of tissue, and not be initiated by a single promoted cell. This makes it seem more like a dynamic phenomenon having to do with cooperation or competition of cells. In this sense, the AAH to BAC transition seems to transcend the simplest Moolgavkar's model.

On the other hand, genetic modifications are observed in the process of AAH and BAC progression. The Fragile Histidine Triad (Fhit) gene at 3p14.2 is a potential tumor suppressor gene and is lost in a number of cancers, including those in lung. The Fhit gene product is a nucleotide binding protein, which may have a role in cell proliferation and apoptotic pathways. Frequent loss of Fhit protein expression and loss of heterozygosity $(\mathrm{LOH})$ at 3 p14.2 have been reported in high-grade dysplasia and squamous carcinoma. $\mathrm{LOH}$ of Fhit reached $43 \%$ in BAC, while Fhit loss is more apparent in more poorly differentiated adenocarcinoma [14]. In view of the definite geometry of the early lesion, it seems doubtful that these changes occur in single precursor cells, which take over the tumor population. So, even a multi-stage carcinogenesis model does not seem applicable here.

In this paper, we aim to understand how cellular dynamics of tumor cells can generate patterns analogous to those phenomenologically observed at the macroscopic scale. We investigate the macroscopic, spatio-temporal effects of the cell cycle with division-rate depending on the concentration of growth factor bound to the cells. Free growth factor particles are transported between cells via diffusion. More sophisticated models of cellular dynamics were developed by Bellomo et al. (see [3,5] and references therein). To obtain model equations they applied the so-called generalised kinetic theory, which provides a statistical description of large cells populations governed by kinetic type interactions. The survey of different models and methods dealing with multiscale modelling of tumor evolution is presented in [4]. The derivation of the macroscopic reaction-diffusion models describing the interplay between the cellular dynamics and the signaling molecules diffusing in the intercellular space has been recently shown in [18] using homogenisation methods of functional analysis. The asymptotic theory proposed by Bellomo and Bellouquid also shows how macroscopic evolution equations can be obtained from the micro-scale description [2].

On the technical level, the objective of this work is to understand the pattern of destabilisation of a population of proliferating cells, assuming a model of cell cycle and a model of binding and surface diffusion of a growth factor. In our analysis, we proceed using diffusion-driven instability (Turing) paradigm. This consists of finding necessary conditions of destabilisation based on the linearised equations and then exploring emerging patterns with the aid of numerical computations.

\section{Model}

In this paper, we explore the version of AAH to BAC transition, which is based on the assumption that initiated (AAH) cells have the ability to proliferate indefinitely, but they still retain dependence on growth factors, which are either externally supplied or may be produced by the cells themselves. The molecules of growth factor, diffuse along the structure formed by the initiated cells and have to bind to cells to stimulate proliferation. We are interested in the response of cells to growth factor under cell cycle regulation, which may lead to fluctuation of cell density and penetration into the surrounding tissue. As evident from the review in the Introduction, the evidence regarding the mechanism of AAH growth and 
AAH to BAC transition is incomplete. Let us summarise our ideas, with emphasis on which elements are supported by observations and which are more speculative in nature:

- We attempt to model growth and invasion process, which leads from a uniform layer of proliferating cells in equilibrium or near-equilibrium (early AAH) to a structure with variable thickness and proliferation rate, which penetrates into the surrounding tissue (late $\mathrm{AAH}$, or BAC).

- In the main body of the paper, we represent the thickness of the cell layer by the surface (or line) cell density; regions of increased density correspond to penetration. In Discussion, we refer to a simple model with explicit thickness geometry and explore its predictions. As mentioned in the review above, increased cell density leads to increased expression of metalloproteases and other factors degrading surrounding tissue.

- Cell proliferation rate is reduced by cell crowding but enhanced in a paracrine manner by a hypothetical growth factor, which may be secreted by the AAH/BAC cells themselves or supplied from the environment and which then diffuses among cells and binds to cell membrane receptors. The main hypothesis of this paper is that the action of this hypothetical growth factor is an explanation for the early invasion patterns observed in $A A H$ and $B A C$.

\subsection{Modelling of cell proliferation}

The model of cell proliferation we use assumes that cells stay in the cell cycle for time, which is a random variable with distribution, the hazard rate of which varies with time and equal to $a(t)$ for cell present in the cycle at time $t$. In addition to this, cells are subject of competing risk $d_{\mathrm{c}}$ of dying while in the cycle. Also, following cell division, each of the progeny cells survives only with probability $p$. Regulation of proliferation is accomplished by modulation of the time variable $a(t)$. This model is by definition stochastic, but the expected values of cell counts may be computed by treating the cell cycle as a well-mixed tank.

The philosophy of building a model of the cell cycle is explained in figure 3: cell cycle is treated as a well-mixed tank, from which cells may either enter division at rate $a(t)$ or die at rate $d_{\mathrm{c}}$ assumed constant in time. The flux to mitosis is equal to $a(t) c(t)$, while the flux to death is equal to $d_{\mathrm{c}} c(t)$. Cells divide with efficiency $p \in[0,1]$, the flux of just divided cells from mitosis back to the cell cycle is equal to $2 p a(t) c(t)$. As a result, the rate of change of $c(t)$ is equal to

$$
\frac{\mathrm{d} c}{\mathrm{~d} t}=2 p a(t) c(t)-a(t) c(t)-d_{\mathrm{c}} c(t) \equiv F(c, b) .
$$

2.1.1 How to model growth regulation? We study two different cases: Model 1. We assume that cell proliferation is regulated by the bound growth factor molecules and depends on the density of the bound growth factor molecules per cell, i.e. $(b(x, t)) /(c(x, t))$ and that this dependence is an increasing function with saturation,

$$
a(c, b)=\frac{a_{0}(b / c)^{m}}{1+(b / c)^{m}}
$$




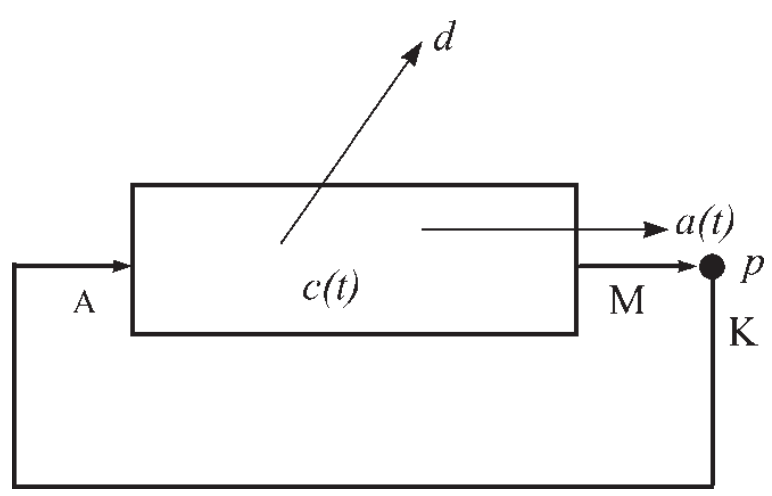

Figure 3. Model of the cell cycle of proliferating cells. A newborn cell enters the cell cycle at point $A$. Cell cycle is treated as a well-mixed tank, from which cells may either enter division at rate $a(x, y, t)$, or death at rate $d$ considered constant in time. As a result, if the number of cells present in the cycle at time $t$ is equal to $c(t)$, the flux to mitosis $(M)$ is equal to $a(x, y, t) c(x, y, t)$, while the flux to death is equal to $d c(x, y, t)$. Cells divide with efficiency $p \in[0,1]$ constant in time. Therefore, the flux of just divided cells from mitosis (point $K$ ) is equal to $2 p a(x, y, t) c(x, y, t)$. These cells constitute the influx at point $A$ of one of progeny cells. Resulting rate of change of $c(x, y, t)$ is equal to $\partial c / \partial t=2 p a c-a c-d c$.

Model 2. We model the proliferation process using a logistic growth function,

$$
a(c, b)=a_{0}(b-K c) \text {. }
$$

Combining equations for $a$ and equation (1) results in the following expression for the kinetics term $F(c, b)$,

$$
F(c, b)=\left[(2 p-1) \frac{a_{0}(b / c)^{m}}{1+(b / c)^{m}}-d_{\mathrm{c}}\right] c,
$$

and

$$
F(c, b)=(2 p-1) a_{0} c(b-K c)-d_{\mathrm{c}} c,
$$

for Models 1 and 2, respectively.

\subsection{Growth factor regulation}

We assume that free growth factor molecules diffuse in the intercellular space. They may be supplied by a hormonal mechanism extraneous with respect to the sheet of tumor cells, or produced by cells. We model it using a function $\kappa(c)$, which is equal to a constant, $\kappa_{0}$, in the case when it is produced by the external medium or is a Menton-Michaelis function of cell density, $\kappa_{0} c(1+c)$, in the case when it is produced by the cells. Molecules of the growth factor bind to receptors on cell's membrane. The number of bound growth factor molecules is denoted by $b$. The rate of the binding depends on the density of the cells and is given by $\alpha(c)$. The function $\alpha(c)$ expresses the density of the sites on the cells to which growth factor molecules can bind (free receptors located on the cell membrane and binding the free growth factor molecules). We assume that the density of such free receptors is a function of cells density. In addition, bound growth factor molecules can dissociate at a rate $d$, and both free and bound growth factor molecules can be eliminated at rates $d_{\mathrm{g}}$ and $d_{\mathrm{b}}$ respectively. 
2.2.1 Transitions in the growth factor molecule space. We assume that cells are embedded in a "field" of growth factor molecules and consider probabilities,

$$
P_{i j}(t)=\operatorname{Pr}[\mathrm{g}(t)=i, \mathrm{~b}(t)=j],
$$

where $\mathrm{g}(t)$ is a number of free growth factor molecules and $\mathrm{b}(t)$ is a number of bound growth factor molecules. Pair $(i, j)$ is the state of the process. Again, all functions of time are also functions of the spatial coordinates $(x, y)$. In a short time interval $(t, t+\Delta t)$ a transition from state $(i, j)$ to state $(k, l)$ occurs with probability $q_{(i, j) \rightarrow(k, l)} \Delta t+o(\Delta t)$. For our model, we specify the following non-zero transition intensities:

$$
\begin{aligned}
& q_{(i, j) \rightarrow(i-1, j+1)}=\alpha(c) i \text { - binding of free growth factor molecules, } \\
& q_{(i, j) \rightarrow(i+1, j)}=\kappa(c) \text {-influx of free growth factor molecules, } \\
& q_{(i, j) \rightarrow(i-1, j)}=d_{\mathrm{g}} i \text {-loss of free growth factor molecules, } \\
& q_{(i, j) \rightarrow(i, j-1)}=d_{\mathrm{b}} \text { - loss of bound growth factor molecules, } \\
& q_{(i, j) \rightarrow(i+1, j-1)}=d j \text {-dissociation of bound growth factor molecules. }
\end{aligned}
$$

A model specified in this way is a time-continuous Markov chain [1]. The chain dynamics are described by the following infinite system of Chapman-Kolmogorov ordinary differential equations for probabilities $P_{i j}$ :

$$
\begin{aligned}
\partial P_{i, j} / \partial t= & \alpha(c)(i+1)\{j \geq 1\} P_{i+1, j-1}+\kappa(c)\{i \geq 1\} P_{i-1, j} \\
& +d_{\mathrm{g}}(i+1) P_{i+1, j}+d_{\mathrm{b}}(j+1) P_{i, j+1}+d(j+1)\{i \geq 1\} P_{i-1, j+1} \\
& -\left(\alpha(c) i+\kappa(c)+d_{\mathrm{g}} i+d_{\mathrm{b}} j+d j\right) P_{i j},
\end{aligned}
$$

where $\{s\} \in\{0,1\}$ is the logical value of statement $s$.

2.2.2 Mean-value equations. Let us define expected counts of free and bound receptor molecules as,

$$
E[g(t)]=\sum_{i, j \geq 0} i P_{i j}(t), \quad E[b(t)]=\sum_{i, j \geq 0} j P_{i j}(t)
$$

Combining equations in (7), we obtain

$$
\dot{b}(t)=\alpha(c) g-d_{\mathrm{b}} b-d b, \quad \dot{g}(t)=-\alpha(c) g-d_{\mathrm{g}} g+\kappa(c)+d b,
$$

where, by an abuse of notations, $\mathrm{g}(t)$ and $\mathrm{b}(t)$ denote expectations $E[\mathrm{~g}(t)]$ and $E[\mathrm{~b}(t)]$, respectively.

\subsection{Final system of equations and hypotheses}

Reasonable hypotheses for functions $a, \alpha$ and $\kappa$ seem to be that

(1) $a$ is an increasing function of the bound growth factor density and a decreasing function of the cell density,

(2) $\alpha$ is an increasing function of cell density, and 
(3) $\kappa$ is either a constant or increasing function of cell density, depending on whether the free growth factor is supplied from outside or is produced by our system's cells, respectively.

In mathematical notation,

$$
\begin{array}{lll}
0 \leq a(b, c) \uparrow \downarrow, & a(0, c)=0, & a(\infty, c)=a_{\infty}(c) \downarrow, \\
& a(b, 0)=a_{0}(b) \uparrow, & a(b, \infty)=0, \\
0 \leq \alpha(c) \uparrow, & \alpha(0)=0, & \\
0 \leq \kappa(c) \uparrow, &
\end{array}
$$

where, in addition, it is assumed that cells do not proliferate in the absence of the growth factor and when they are crowded to the extreme, as well as that no growth factor binding is possible in the absence of cells. These assumptions are satisfied by Models 1 and 2.

We consider two geometries:

Geometry 1. Line of cells, occupying the interval $x \in[0, L]$, There are three substances distributed over the line's length: cells, free and bound growth factor molecules, with densities $\mathrm{c}(x, t), \mathrm{g}(x, t)$ and $\mathrm{b}(x, t)$, respectively.

Geometry 2. Thin sheet of cells, occupying the square $(x, y) \in\left[0, L_{1}\right] \times\left[0, L_{2}\right]$, is rolled to form a cylindrical tube such that the intervals $\left\{(x, 0), x \in\left[0, L_{1}\right]\right\}$, and $\{(x$, $\left.\left.L_{2}\right), x \in\left[0, L_{1}\right]\right\}$ coincide. There are three substances distributed over the cylinder's surface: cells, and free and bound growth factor molecules, with densities $\mathrm{c}(x, y, t), \mathrm{g}(x, y$, $t)$ and $\mathrm{b}(x, y, t)$, respectively.

Diffusion. It is assumed that free growth factor molecules can diffuse over the surface of the tube or length of the line, with diffusion coefficient $D_{\mathrm{g}}$.

For both geometries, model equations have the form,

$$
\begin{aligned}
& \frac{\partial c}{\partial t}=\left((2 p-1) a(b, c)-d_{\mathrm{c}}\right) c, \\
& \frac{\partial b}{\partial t}=\alpha(c) g-d_{\mathrm{b}} b-\mathrm{d} b, \\
& \frac{\partial g}{\partial t}=D_{\mathrm{g}} \Delta g-\alpha(c) g-d_{\mathrm{g}} g+\kappa(c)+d b,
\end{aligned}
$$

with homogeneous Neumann (zero-flux) boundary conditions for $g$ in case of Geometry 1 ,

$$
\partial_{x} g(0, t)=\partial_{x} g(1, t)=0 ; \quad x \in[0, L],
$$

and mixed zero-flux and periodic boundary conditions in case of Geometry 2 ,

$$
\partial_{x} g(0, y, t)=\partial_{x} g\left(L_{1}, y, t\right)=0 ; \quad y \in\left[0, L_{2}\right], \quad g(x, 0, t)=g\left(x, L_{2}, t\right) ; \quad x \in\left[0, L_{1}\right], \quad t \geq 0 .
$$

Some of the analysis carried out in this work is equally valid for both geometries. As a rule, we consider the simpler Geometry 1, and then discuss extension to Geometry 2. 
Accordingly, consider now Geometry 1 . After rescalling of the variable $x$, we obtain,

$$
\begin{aligned}
& \frac{\partial c}{\partial t}=\left((2 p-1) a(b, c)-d_{\mathrm{c}}\right) c, \\
& \frac{\partial b}{\partial t}=\alpha(c) g-d_{\mathrm{b}} b-\mathrm{d} b, \\
& \frac{\partial g}{\partial t}=\frac{1}{\gamma} \Delta g-\alpha(c) g-d_{\mathrm{g}} g+\kappa(c)+d b,
\end{aligned}
$$

with homogeneous Neumann (zero flux) boundary conditions for $g$,

$$
\partial_{x} g(0, t)=\partial_{x} g(1, t)=0 ; \quad x \in[0,1],
$$

Coefficient $1 / \gamma$ is a composite parameter including the diffusion constant and scalling parameters, $\gamma=L^{2} / d$.

\section{Existence and number of space-homogeneous steady states}

Equations for the space-homogeneous steady states are obtained by setting all the derivatives equal to 0 in the model equations,

$$
\begin{gathered}
c\left[(2 p-1) a(b, c)-d_{\mathrm{c}}\right]=0, \\
\alpha(c) g-d_{\mathrm{b}} b-d b=0, \\
-\alpha(c) g-d_{\mathrm{g}} g+d b+\kappa(c)=0 .
\end{gathered}
$$

From equation (13) we obtain,

$$
\bar{g}=\frac{\kappa(\bar{c})+d \bar{b}}{\alpha(\bar{c})+d_{\mathrm{g}}}
$$

which, after substitution into equation (12), yields

$$
\bar{b}=\frac{\alpha(\bar{c}) \kappa(\bar{c})}{d d_{\mathrm{g}}+d_{\mathrm{b}}\left[\alpha(\bar{c})+d_{\mathrm{g}}\right]} .
$$

The trivial steady state with $c=0$ can be analysed separately. If we exclude it, we obtain that $\bar{c}$ has to satisfy the following equation

$$
a\left\{\frac{\alpha(\bar{c}) \kappa(\bar{c})}{d d_{\mathrm{g}}+d_{\mathrm{b}}\left[\alpha(\bar{c})+d_{\mathrm{g}}\right]}, \bar{c}\right\}-\frac{d_{\mathrm{c}}}{2 p-1}=0 .
$$

Let us note that function $a$ is increasing in the first argument and decreasing in the second, while both arguments themselves are increasing in $\bar{c}$. This implies that existence and number of solutions may be difficult to determine and depends on the particular form of functions $a$, $\alpha$ and $\kappa$. 
In numerical computations, we will consider the following parametric forms of functions $\alpha$ and $\kappa$,

$$
\begin{aligned}
& \alpha(c)=\alpha_{0} c^{s}, \quad \alpha_{0}, s \geq 1, \\
& \kappa(c)=\kappa_{0} \frac{c}{c+1} \quad \text { or } \\
& \kappa(c)=\kappa_{0}, \quad \kappa_{0} \geq 0 .
\end{aligned}
$$

In next section, we derive restrictions on the kinetics of equation (10), which allow the diffusion-driven destablisation of the spatially homogeneous stationary solutions and pattern formation. Then, for the particular forms of the functions $a, \alpha$ and $\kappa$ we find the space of parameters, for which such positive steady states exist.

\subsection{Existence, positivity and boundedness}

Using the framework of invariant rectangles (see [22] for $\Omega \subset \mathbb{R}^{1}$, [7] for $\Omega \subset \mathbb{R}^{n}$ ) we can show that solutions of system (10) remain uniformly bounded and positive for positive initial conditions. The existence of global solutions results from a standard argument based on the theory of bounded invariant rectangles and a priori estimates [22]. Methods outlined in [22, Chapter 14], can be used without major modifications.

\section{Transient dynamics of the models}

\subsection{Diffusion driven instabilities in the three equations model with one diffusion operator}

In this section, we look for the diffusion-driven mechanism for pattern formation. Diffusiondriven instability (Turing-type instability) arises in a reaction-diffusion system and uses special features of diffusion, which results in destabilisation of the spatially homogeneous steady state. It occurs when there exists a spatially homogeneous solution, which is asymptotically stable in the sense of linearised stability in the space of constant functions, but is unstable with respect to spatially inhomogeneous perturbations. In models with such property patterns can arise spontaneously.

For the study of coupled system of reaction-diffusion equations and ordinary differential equations it is convenient to consider stationary solutions $\bar{c} \in L^{\infty}(\Omega), \bar{g}, \bar{b} \in H^{2}(\Omega)$. It means that first two equations (ODEs) hold almost everywhere in $\Omega$ while the third equation (a reaction-diffusion equation) holds in the usual weak sense.

We consider bifurcation from the steady state by examining the response of the system to an initially small perturbation from the steady value $(\bar{c}, \bar{b}, \bar{g})$. Stability of smooth stationary solutions of reaction-diffusion equations may be analysed by considering the eigenvalues of the linearised system. We carry out a linear stability analysis to derive the conditions under which instability can arise (for details see, for example, [9,20]). We apply the spectral criterion of linearised stability. A spectral criterion for reaction-diffusion equations coupled with ODEs was developed in [16]. It is a modified version of the theorem of Henry ([10], Theorem 5.1.1).

Linear stability analysis of system (10) around a spatially homogenous steady state allows us to formulate necessary and sufficient conditions for the diffusion-driven patterns in the three-variable model with one diffusion operator in the third equation. 
Lemma 4.1. Let $A$ be the Jacobian matrix computed at a positive spatially homogeneous steady state. There is a diffusion-driven instability for system (10) if and only if the following conditions are fulfilled:

$$
\begin{gathered}
-\operatorname{tr}(A)>0, \\
-\operatorname{tr}(A) \sum_{i<j}\left|A_{i j}\right|+|A|>0, \\
-|A|>0, \\
\left|A_{12}\right|<0,
\end{gathered}
$$

where $A_{i j}$ is a submatrix of $A$ consisting of the $i$-th and $j$-th column and $i$-th and $j$-th row, and $|A|$ and $\left|A_{i j}\right|$ denote the determinants of matrices $A$ and $A_{i j}$, respectively.

Details of the linear stability analysis are presented in the Appendix.

\subsection{Destabilisation conditions for Models 1 and 2}

Now we have to verify whether model (10) fulfills the conditions for diffusion-driven destabilisation. We calculate the Jacobian matrix $A$ at a positive steady state,

$$
a(\bar{b}, \bar{c})=d_{\mathrm{c}}, \quad \bar{g}=\frac{\kappa(\bar{c})-d_{\mathrm{b}} \bar{b}}{d_{\mathrm{g}}}, \quad \alpha(\bar{c}) \bar{g}=\left(d_{\mathrm{b}}+d\right) \bar{b},
$$

and obtain,

$$
A=\left(\begin{array}{lll}
\frac{\partial}{\partial c} a(\bar{b}, c) \bar{c} & \frac{\partial}{\partial b} a(\bar{b}, c) \bar{c} & 0 \\
\alpha^{\prime}(\bar{c}) \bar{g} & -\left(d_{\mathrm{b}}+d\right) & \alpha(\bar{c}) \\
-\alpha^{\prime}(\bar{c}) \bar{g}+\kappa^{\prime}(\bar{c}) & d & -\alpha(\bar{c})-d_{\mathrm{g}}
\end{array}\right)
$$

Calculating $\left|A_{12}\right|=a_{11} a_{22}-a_{12} a_{21}$ using the steady states conditions, (18), we conclude that $\left|A_{12}\right|<0$ if and only if

$$
\left(-\left.\frac{\partial a}{\partial c}\right|_{(\bar{b}, \bar{c})} \bar{c}\right) \alpha(\bar{c})<\left.\left(\left.\frac{\partial a}{\partial b}\right|_{(\bar{b}, \bar{c})} \bar{b}\right) \alpha^{\prime}(c)\right|_{\bar{c}} \bar{c} .
$$

First of all, if function of proliferation rate, $a$, is a function increasing in $c$, then $a_{11}>0$ and condition (19) is automatically fulfilled. However, in this paper, we do not consider the case of such self-enhancement in proliferation, which leads to the unbounded growth of cell density. We notice only that, in case of the model of one ODE coupled with a reactiondiffusion equation, the requirement that $a_{11}>0$ is a necessary condition for the diffusiondriven pattern formation mechanism [17]. In case of the higher dimension of the ODEs 
subsystem, self-enhancement in the proliferation rate is not any more demanded. Here, we study two examples of the different type of the function describing the proliferation rate.

Model 1. Assuming that $a=a(b / c)$ we obtain that

$$
-\left.\frac{\partial}{\partial c} a\right|_{(\bar{b}, \bar{c})} \bar{c}=\left.\frac{\partial}{\partial b} a\right|_{(\bar{b}, \bar{c})} \bar{b}
$$

We conclude that

Lemma 4.2. There is a diffusion-driven instability for the system (10) with $a=a(b / c)$ if and only if

$$
\alpha(\bar{c})<\bar{c} \frac{\partial}{\partial c} \alpha(\bar{c})
$$

in a steady state $\bar{c}$.

Therefore, diffusion-driven pattern formation can occur if

$$
\alpha(c)=\alpha_{1} c^{s+1}, \quad s>0,
$$

i.e. process of binding free growth factor particles to cells is very fast. Further, we assume that $a$ is an increasing function with saturation,

$$
a=\frac{a_{0}(b / c)^{m}}{1+(b / c)^{m}}
$$

Using equation (18) we calculate that a homogeneous positive steady state $(\bar{c}, \bar{b}, \bar{g})$ must fulfill the following implicit relationship,

$$
\begin{gathered}
\frac{\bar{b}}{\bar{c}}=\left\{\frac{d_{\mathrm{c}}}{a_{1}-d_{\mathrm{c}}}\right\}^{1 / m}=: X, \\
\bar{g}=\left(d+d_{\mathrm{b}}\right) \frac{\bar{b}}{\alpha(\bar{c})}
\end{gathered}
$$

and

$$
\kappa(\bar{c})=d_{\mathrm{b}} X c+d_{\mathrm{g}}\left(d+d_{\mathrm{b}}\right) X \frac{\bar{c}}{\alpha(\bar{c})}
$$

From equation (24) we immediately conclude that the model has positive steady states only if $a_{1}>d_{\mathrm{c}}$. Furthermore, since, for $\alpha$ given by equation (20), the right-hand side of equation (24) is a convex function, for both choices of a function $\kappa(\kappa(c)=\kappa$ and $\kappa(c)=\kappa(c /(1+c)))$ we can choose parameter value $\kappa$ in such way that equation (24) has 
two positive solutions. For constant $\kappa$ we calculate that for

$$
\kappa>2 X \sqrt{\frac{d_{\mathrm{b}} d_{\mathrm{g}}\left(d+d_{\mathrm{b}}\right)}{\alpha}}
$$

we always have two positive solutions of equation (24).

Model 2. Now, we consider the case when $\alpha(c)$ is a linear function of $c$ and binding of free growth factor particles to cells is described by $\alpha_{0} c g$. In this case, requirement (19) is fulfilled if

$$
-\left.\frac{\partial}{\partial c} a\right|_{(\bar{b}, \bar{c})} \bar{c}<\left.\frac{\partial}{\partial b} a\right|_{(\bar{b}, \bar{c})} \bar{b} .
$$

The above condition is satisfied by a logistic type of proliferation function, namely $a(c, b)=$ $a_{1}(b-K c)$ (which means that the proliferation kinetics is given by $\left.a_{1}(b-K c) c\right)$. Here, the proliferation rate is limited by availability of bound growth factor particles.

For Model 2, using equation (18) we find the requirements for the homogeneous positive steady state $(\bar{c}, \bar{b}, \bar{g})$,

$$
\bar{b}-K \bar{c}=\frac{d_{\mathrm{c}}}{a_{1}}, \quad \bar{g}=\left(d+d_{\mathrm{b}}\right) \frac{\bar{b}}{\alpha_{0} \bar{c}} .
$$

and

$$
\kappa(\bar{c})=d_{\mathrm{b}} K \bar{c}+\frac{d_{\mathrm{b}} d_{\mathrm{c}}}{a_{1}}+\frac{d_{\mathrm{g}}\left(d+d_{\mathrm{b}}\right) K}{\alpha_{0}}+\frac{d_{\mathrm{g}} d_{\mathrm{c}}\left(d+d_{\mathrm{b}}\right)}{a_{1} \alpha_{0} \bar{c}} .
$$

We conclude that there exists a region of values of $\kappa$ such that (26) has two positive solutions and, therefore, there exist two positive steady states for Model 2.

\subsection{Dispersion relation}

Let us have a closer look at the dispersion relation, which we obtain for the model (10). From the proof of Lemma 8.1 we observe that the destabilisation of the homogeneous steady state is possible if and only if

$$
|\tilde{A}|=|A|-\left|A_{12}\right| \frac{\mu_{m}^{2}}{\gamma}>0 .
$$

Condition (27) is verified if $\mu_{m}^{2} / \gamma$ is large enough. As a consequence, for every $\gamma$, there exist infinitely many different integer $\mu_{\mathrm{m}}$ for which the above inequality is fulfilled. It means that there is an infinite range of unstable modes. The dispersion relation, $\lambda=\lambda\left(\mu_{m}^{2} / \gamma\right)$, which is an algebraic equation for the growth rate, is shown in figure 4. Asymptotic analysis of systems with such dispersion relation seems to be an open problem. It is fundamentally different from the typical dispersion relation, obtained for example in models of two diffusive chemicals [20]. Such best-known dispersion relation determines the bounded range of unstable modes, the size of which depends on the bifurcation parameter $\gamma$. Then a certain range of $\gamma$ can be determined for which there exists only one unstable mode. It allows to select, by changing the scalling parameter $\gamma$, the mode, which grows to the long-term 


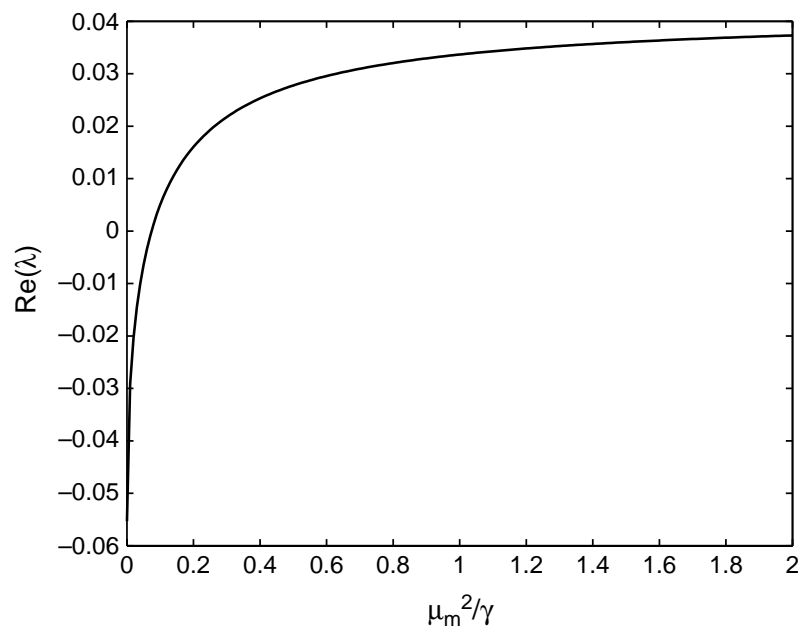

Figure 4. Dispersion relation $\lambda=\lambda\left(\mu_{m}^{2} / \gamma\right)$ for the model (10) It shows that for a given $\gamma$ there exists an infinite range of unstable modes, i.e. $\operatorname{Re}(\lambda)>0$ for infinitely many $\mu_{m}^{2}$. A single unstable mode cannot be selected.

heterogeneous pattern. If the range of unstable modes is finite but includes more values then there is a competition between these patterning modes.

In our model, similar to the 2-equation model of reference [17], it is impossible to select a single unstable mode. In other words, from the dispersion relation we cannot decide which eigenfunctions, that is, which spatial patterns, are linearly unstable and grow with time. There does not exist a wavenumber with maximum $\operatorname{Re}(\lambda) . \operatorname{Re}(\lambda)$ grows monotonously with $\mu_{m}^{2} / \gamma$. However, we can show that $\operatorname{Re}(\lambda)$ is bounded from above.

Simulations show that the index of the growing mode depends on initial conditions and on the scalling parameter $\gamma$ but in rather different manner than in the model consisting only of one ODE and one reaction-diffusion equations as studied in [17] (see figures 6 and 7 and discussion in section 5.1.2).

\section{Numerical results}

We performed simulations for the models with one- and two-dimensional structures. Since the results look qualitatively the same, for clarity we present the results for the onedimensional model. We consider particular forms of Models 1 and 2. For Model 1 we assume that

$$
\alpha(c)=\alpha c^{2}, \quad a(b, c)=(2 p-1) \frac{a_{0}(b / c)^{m}}{1+(b / c)^{m}} .
$$

For Model 2 we assume,

$$
\alpha(c)=\alpha c, \quad a(b, c)=(2 p-1) a_{0}(b-K c) .
$$

For both models we perform numerical simulations for constant $\kappa$, which corresponds to the extraneous source of new growth factor particles, as well as for $\kappa(c)=\kappa_{0}(c /(1+c))$, which 
corresponds to the synthesis of growth factor by cells. This particular choice of function $\kappa$ does not change the qualitative results of the simulations.

As discussed in section 4.2, in both models, Models 1 and 2, we can choose the parameter $\kappa$, for which the system has two positive spatially homogeneous steady states, one of which fulfills diffusion-driven instability conditions (14)-(17). In the simulations, such steady state is perturbed using small random or cosine additive terms with different frequencies (see figure 5). Simulations show that spatially uniform initial data evolve into a spatially inhomogeneous pattern of spikes. The qualitative behavior of solutions for different variants of the model is similar in the whole set of admissible parameters.

\subsection{Data}

Cell kinetics. For computations, we assume that the expected interdivision time of cells is equal to $1 / a_{0}=12 \mathrm{~h}$ similar to the estimates in [15]. Further, we assume a perfect efficiency of divisions $p=1$, and a low death rate $d_{\mathrm{c}}$. We performed simulations for different values of $d_{\mathrm{c}}$ adjusting the value of $\kappa$ accordingly.

Growth factors kinetics. We assume the expected turnover time equal to $1 / d_{\mathrm{b}}=1 / d_{\mathrm{g}}=10 \mathrm{~h}$. Also, we use arbitrary values of $\alpha_{0}=0.1$ or $\alpha_{0}=1$ and $d=0.1$. Coefficient $\kappa$ is adjusted to ensure existence of two positive steady states. Therefore, parameters used in simulations satisfy necessary conditions for diffusion driven instability. We performed a series of simulations of Model 1 with different $\alpha_{0}$ and different values of the constant function $\kappa(\kappa=3, \kappa=10, \kappa=0.5)$ as well as $\kappa(c)=\kappa_{0}(c /(1+c))$. Numerical solutions of the models exhibit similar trends in dynamics. Therefore, to illustrate the dependence of solutions on the diffusion rate and initial perturbation, we focus our attention on Model 1 with constant $\kappa$ and a particular parameter set: $a_{1}=1 / 12, d_{\mathrm{c}}=0.05$, $d_{\mathrm{b}}=d_{\mathrm{g}}=0.1, \kappa=0.5, m=1$ and $\alpha=0.1$.
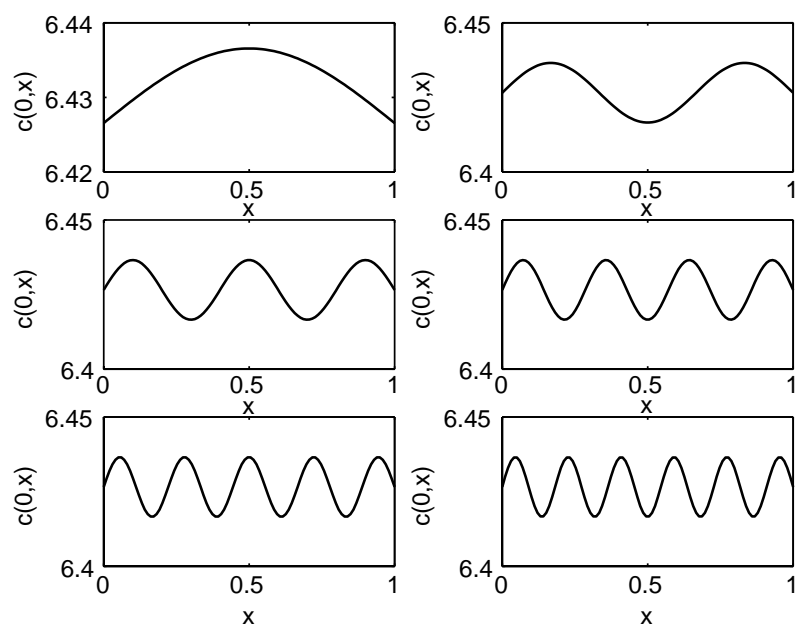

Figure 5. Different initial functions used in numerical simulations, $c(x, 0)=\bar{c}+\varepsilon \cos (n \pi x-\pi / 2)$, where $\bar{c}$ is a spatially homogeneous steady state, $\varepsilon$ is a size of a small initial perturbation (in presented further results of simulations $\epsilon$ was set to 0.01 . Changing the value of $\varepsilon$ leads to lengthening of the period of the near-equilibrium observed in simulations. 



Figure 6. Time evolution of solutions of Model 1 with constant growth factor particles synthesis $\kappa$ and parameters: $a_{1}=1 / 12, d_{\mathrm{c}}=0.05, d_{\mathrm{b}}=d_{\mathrm{g}}=0.1, \kappa=0.5, m=1, \alpha=0.1$. On the left-hand side we present solution $c(x, t)$, in the centre $b(x, t)$, and on the right-hand side $g(x, t)$. Rows 1,2 and 3 correspond to $\gamma=1, \gamma=10$ and $\gamma=100$, respectively. Initial perturbation of the steady state value is cosine function with one maximum (see figure 4) and amplitude 0.01. We observe formation of spike patterns with the number of spikes dependent on the scalling coefficient $\gamma$. When $\gamma$ increases (what corresponds to the decreasing diffusion rate), the minimal unstable mode $\mu_{\mathrm{m}}$ increases (compare with figure 4 of dispersion relation) and the number of spikes in final pattern increases.

5.1.1 Rate of increase of spike solutions. As evident from figures 6-9 the complete system exhibits a period of near-equilibrium, i.e. cell density $c(x, t)$ remains close to $c(x, 0)$ until a threshold time at which it starts exponentially increasing at some $x$-coordinates and exponentially decreasing at other. The rate of the exponential growth read from the graph is consistent with the eigenvalue $\lambda\left(\mu_{m}^{2} / \gamma\right)$ corresponding to the minimum wavenumber $\mu_{\mathrm{m}}$ for which the destablisation occurs, i.e. for which $\lambda\left(\mu_{m}^{2} / \gamma\right)$ is positive. The solutions stay uniformly bounded and after some period of growth the height of peaks (spikes) reaches a constant. Numerical calculation also indicates that the length of the dormancy period can be calculated from the linearised system and depends on the amplitude of the initial perturbation. Assume that the solution of our model close to the homogeneous steady state is of the form, $c(x, t)=\bar{c}+\varepsilon \mathrm{e}^{\lambda_{1} t} \phi_{m}(x)$, where $\lambda_{1}$ corresponds to the growing mode. Using this conjecture, we can calculate for which values of $t$ the solution stays close to the steady state $\bar{c}$. Calculated $\lambda_{1}$ and $t=\left(1 / \lambda_{1}\right) \ln (1 / \varepsilon)$ are consistent with the initial spike growth rate and the length of the dormancy period as read from the graphs.

5.1.2 Spatial profiles of solutions: Dependence on diffusion coefficient. Series of simulations, depicted in figures 6-9, show that the destabilisation of a spatially homogeneous steady state leads to the formation of spikes. At the very beginning the 



Figure 7. Time evolution of the solutions $c(x, t)$ of Model 1 with the parameter set as in Figure 6, scalling coefficient $\gamma=1$ and initial perturbations with cosine functions of different frequencies (with different number of local maxima) shown in Figure 5. We can see that the number of growing spikes corresponds to the minimal unstable wavenumber $\mu_{\mathrm{m}}=1$ when the initial perturbation has odd number of maxima. In case of even number of maxima in the initial function, we observe formation of two spikes.

number of the growing spikes reveals the number of maxima in the initial perturbation. However, after a short time some of them may disappear and the final pattern consists of the number of spikes corresponding to the minimum $\mu_{\mathrm{m}}$ for which destabilisation occur. Therefore, the spatial shape of final patterns depends on parameter $\gamma$, the inverse of the diffusion coefficient (compare with the dispersion relation shown in figure 4). For $\gamma$ small enough we may observe a pattern of several or even only one spike. When $\gamma$ increases, so does the number of spikes. When $\gamma$ increases, the solution profile displays an increasing number of local maxima. For a regular initial perturbation (with cosine function) the final pattern is also regular in the sense of similar shapes of spikes and symmetries, while for a random initial perturbation, a chaotic-like profile emerges. For large $\gamma$, we observe patterns with many spikes of different heights, which even in the case of regular initial perturbation look apparently chaotic (see figure 8). In such case, the number of spikes does not reveal anymore the minimum wavenumber estimated from the dispersion relation; this might be an effect of discretisation. We see that the higher-order wave numbers are damped in the numerical discretisation. The number of spikes is equal to the smallest unstable mode, which can be calculated from the dispersion relation for the linearised system. However, it also depends on the symmetry of the initial perturbation. In case of the symmetric initial perturbation with an even number of local maxima, the final pattern has an even number of spikes, even if the smaller odd number is already unstable (see figure 7). 
$[\mathrm{A}]$

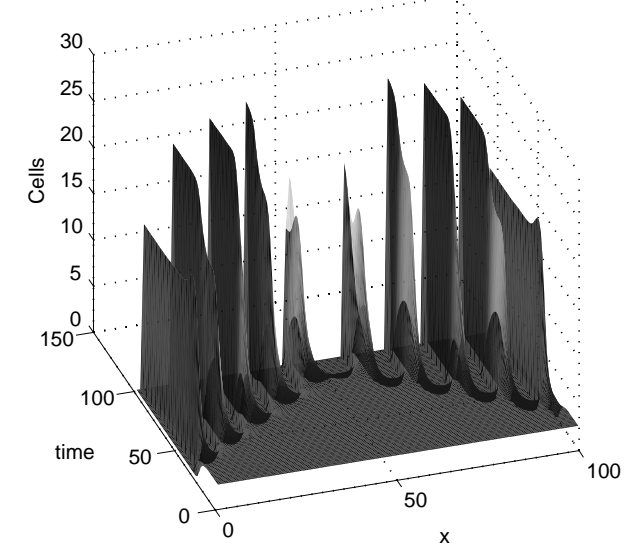

$[B]$

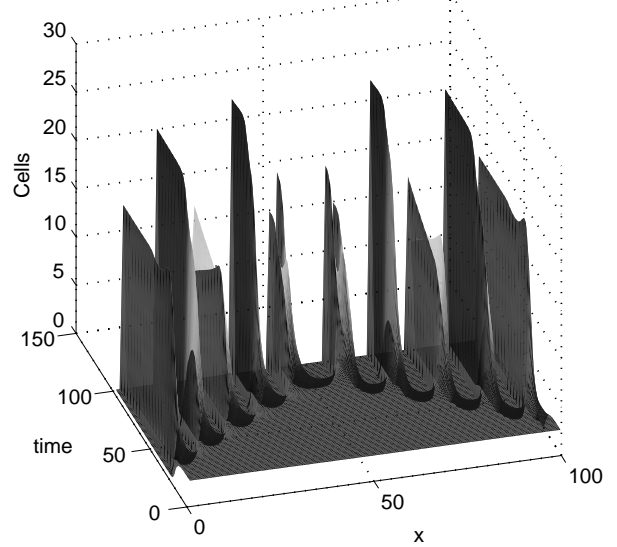

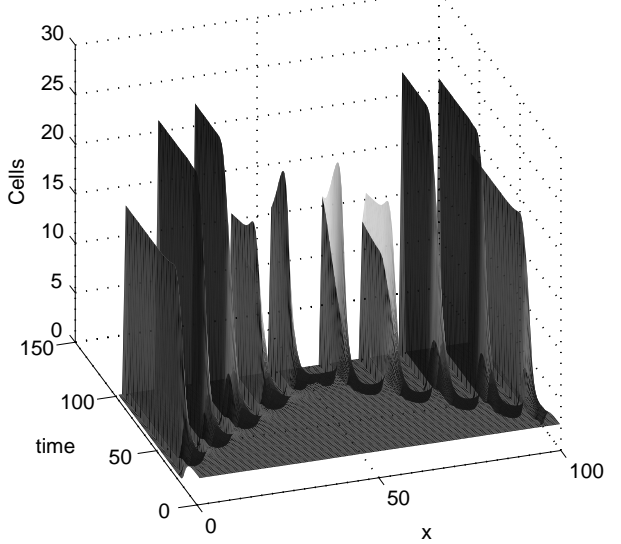

Figure 8. Numerical simulations of Model 1 with the parameter set as in Figure 6 and a large scalling parameter $\gamma=50,000$. The simulations were performed for the initial perturbation of the spatially homogeneous steady state with different cosine functions. 

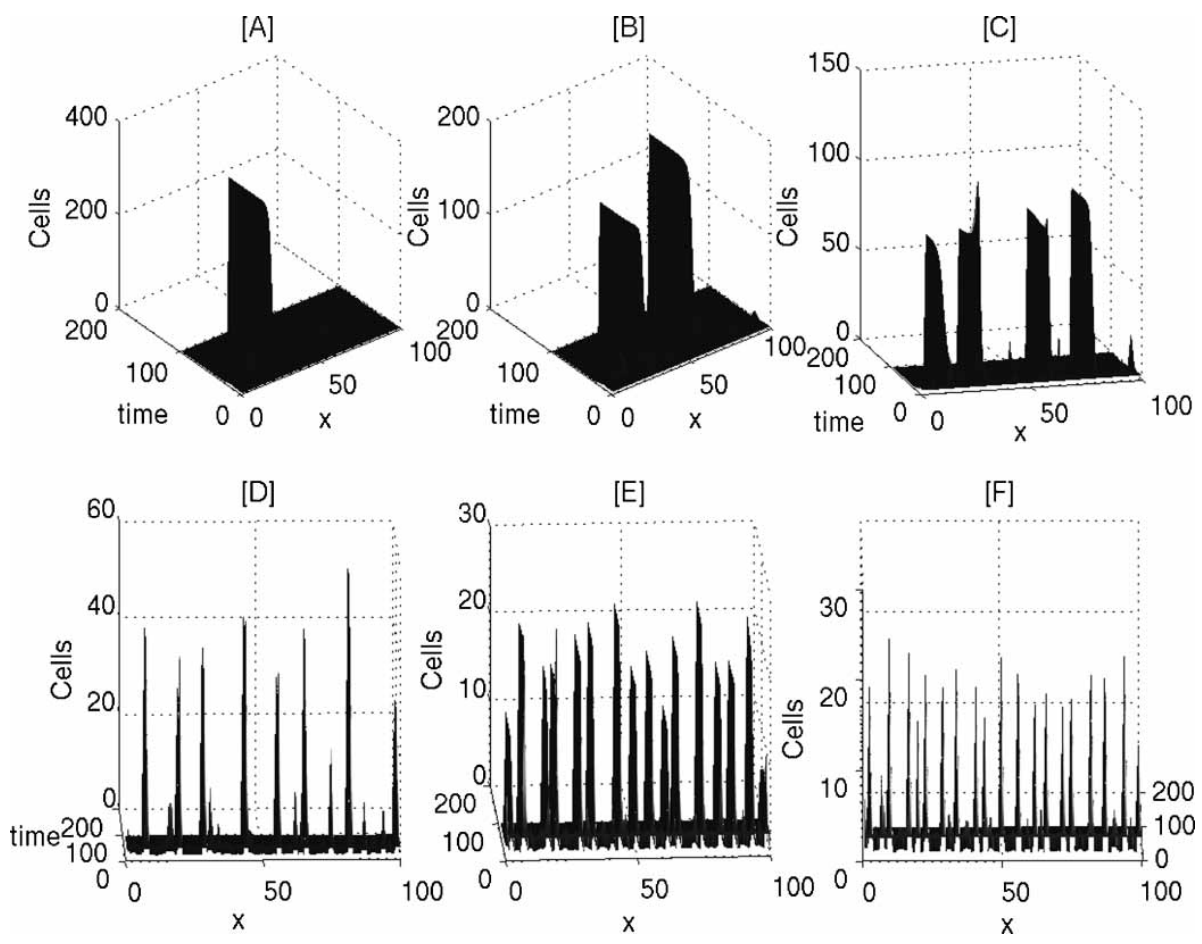

Figure 9. Numerical simulations of Model 1 with the parameter set as in Figure 6, and a range of scalling parameters $\gamma=10, \gamma=1, \gamma=10, \gamma=100, \gamma=1000, \gamma=10,000$. The simulations were performed for the random initial perturbation of the spatially homogeneous steady state.

\section{Discussion}

The model of early cancer invasion developed in this paper assumes that cell proliferation rate is reduced by cell crowding but enhanced in a paracrine manner by a hypothetical growth factor, which may be secreted by the AAH/BAC cells themselves or supplied from the environment and which then diffuses among cells and binds to cell membrane receptors. As stated in the Introduction, the main hypothesis of this paper is that the action of this hypothetical growth factor is an explanation of the early invasion patterns observed in $A A H$ and BAC. As detailed in the Results section, if the reaction-diffusion system we consider has parameter values inside the region of Turing pattern formation regime, the patterns emerging have the form of spikes, the number and relative arrangement of which strongly depend on the strength of diffusion and initial small perturbation of the spacehomogeneous steady state. These spike solutions seem to be very much like the protrusions into the surrounding tissue matrix, observed in AAH and BAC (figure 2, panels $\mathrm{C}$ and $\mathrm{D}$ ).

It is worth noticing that this dynamics, based on two ODEs coupled with one reactiondiffusion equation, differs from the dynamics observed in a model involving one ODE coupled with one reaction-diffusion equation (compare [17]). In this former model, the final pattern depended more on the initial perturbation than on the scalling coefficient $\gamma$. Even for small values of $\gamma$, corresponding to the existence of small unstable modes, we observed formation of the spatial structures, which strongly resembled initial conditions, i.e. had the 
same number of maxima. When $\gamma$ increased, the solution profile loses its similarity to the initial condition and displays an increasing number of the local maxima. Moreover, in the current model we observe spike solutions, while in the models with only one ODE we observed the growth of "broader humps". For the large $\gamma$ we observed similar dynamics as in the 3-equation models discussed here. The difference in the pattern structure may stem from different number of ODEs in the current model as compared to the model of reference [17].

In our model, free growth factor molecules diffuse in the extracellular space while binding processes take place at the cells membrane. Recently in [18], using multi-scale analysis one of us showed that such receptor-ligand binding processes can be modeled by the reactiondiffusion equations coupled with the ODEs in case all the membrane processes are homogeneous within the membrane. The framework we consider in the current paper, seems to be applicable to many processes, which result from the interplay between intra-cell dynamics and inter-cell signaling. In this sense, our model seems able to be applied to different biological setups.

\subsection{Effects of geometry and transport}

As already discussed, the patterns of local invasion have been obtained by modelling spikes of the one- or two-dimensional cell density function. No physical nor geometric model for invasion was considered. To understand the importance of geometry, we may consider the system with transport (local invasion) of cells along an additional spatial coordinate called $z$. Now $c=c(t, x, z)$ and so forth. To free ourselves of other mechanisms and to radically simplify the analysis, we assume that the processes of production and binding of growth factor molecules are at equilibrium and there is no diffusion. We also neglect growth factor dissociation from cells. Then we obtain

$$
\begin{aligned}
& \frac{\partial c}{\partial t}+q \frac{\partial c}{\partial z}=c\left[(2 p-1) a(b, c)-d_{\mathrm{c}}\right] ; c(t, 0)=c_{0}(t) \\
& 0=\alpha(c) g-d_{\mathrm{b}} b \\
& 0=-\alpha(c) g-d_{\mathrm{g}} g+\kappa(c),
\end{aligned}
$$

where $q$ is the rate of invasion, which is considered constant. Solving the second and third equation (both being algebraic) and substituting into the first equation, we obtain

$$
\frac{\partial c}{\partial t}+q \frac{\partial c}{\partial z}=q r(c)
$$

where

$$
q r(c)=a\left\{\frac{\alpha(c) \kappa(c)}{d_{\mathrm{b}}\left[\alpha(c)+d_{\mathrm{g}}\right]}, c\right\}-\frac{d_{\mathrm{c}}}{2 p-1} .
$$

Let us assume now that $a\left\{\alpha(c) \kappa(c) /\left\{d_{\mathrm{b}}\left[\alpha(c)+d_{\mathrm{g}}\right]\right\}, c\right\}$ has the property of being equal to 0 at $c=0$, converging to 0 as $t \rightarrow \infty$, and being positive with a single maximum for $c \in(0, \infty)$. Then, $1 / r(c)$ has two hyperbola-type singularities at the points at which $r(c)$ has zeros.

Returning to the equation, we see that the characteristics have the form of

$$
z\left(t, t_{0}\right)=q\left(t-t_{0}\right) ; \quad t \geq t_{0} .
$$


The solution along characteristics

$$
\tilde{c}\left(t, t_{0}\right)= \begin{cases}c\left(t, q\left(t-t_{0}\right)\right) & t>t_{0} \\ c_{0}(t) & t=t_{0}\end{cases}
$$

satisfies the equation

$$
\frac{\mathrm{d} \tilde{c}}{\mathrm{~d} t}=\operatorname{qr}(\tilde{c})
$$

which has the solution implicitly given by

$$
\int_{c_{0}(t)}^{\tilde{c}\left(t, t_{0}\right)} \frac{\mathrm{d} x}{r(x)}=q\left(t-t_{0}\right)
$$

which can be written as

$$
R\left[\tilde{c}\left(t, t_{0}\right)\right]-R\left[c_{0}\left(t_{0}\right)\right]=q\left(t-t_{0}\right),
$$

where function $R$ can be evaluated only outside the singularities of $1 / r(c)$. Wherever $R$ is defined

$$
\tilde{c}\left(t, t_{0}\right)=R^{-1}\left\{R\left[c_{0}\left(t_{0}\right)\right]+q\left(t-t_{0}\right)\right\} .
$$

Expressing the latter in the terms of $c(t, z)$, we obtain

$$
c(t, z)=R^{-1}\left\{R [ c _ { 0 } ( t - z / q ) + z \} \stackrel { t \rightarrow \infty } { \longrightarrow } R ^ { - 1 } \left\{R\left[c_{0}(\infty)+z\right\} .\right.\right.
$$

The interpretation is that the function $c(t, z)$, for any given $z$, increases with time. This is equivalent to invasion. However, even at infinite times, $c(\infty, z)$ is nontrivially dependent of $z$. The density of cells increases with $z$ (assuming the initial concentration $c_{0}$ is located above the lower singular point) and reaches saturation level at infinite $z$. This implies that the total mass of invading cells increases indefinitely. Let us notice that with the transport term absent, the total mass of cells stabilises. Therefore, taking into account transport, an independent coordinate may radically alter the form and behavior of solutions. This question seems worth pursuing.

\section{Acknowledgements}

We would like to thank Dr Keith Kerr for explaining to us current views on early lung cancer progression. Marek Kimmel was supported by NCI grant CA097431. Anna MarciniakCzochra was supported by Center for Modeling and Simulation in the Biosciences (BIOMS) in Heidelberg.

\section{References}

[1] Anderson, W.J., 1991, Continuous Time Markov Chains: An Applications-oriented Approach (New York, NY: Springer-Verlag). 
[2] Bellomo, N. and Bellouquid, A, 2004, From a class of kinetic models to macroscopic equations for multicellular systems in biology, Discrete and Continous Dynamical Systems, 4, 59-80.

[3] Bellomo, N., Bellouquid, A and Delitala, M, 2004, Mathematical topics on the modelling of multicellular systems in the competition between tumor and immune cells, Mathematical Models and Methods in Applied Sciences, 14, 1683-1733.

[4] Bellomo, N., De Angelis, E. and Preziosi, L., 2003, Multiscale modelling and mathematical problems related to tumor evolution and medical therapy, Journal of Theoretical Medicine, 5, 111-136.

[5] Bellouquid, A. and Delitala, M., 2005, Mathematical methods and tools of kinetic theory towards modelling complex biological systems, Mathematical Models and Methods in Applied Sciences, 15, 1639-1666.

[6] Burns, D.M., Major, J.M. and Shangs, T.G., 2001, Risks associated with smoking cigarettes with low machinemeasured yields of tar and nicotine, Monograph 13, in Smoking and Tobacco Control. National Cancer Institute, Washington DC, USA, http://cancercontrol.cancer.gov/tcrb/Monographs/13/.

[7] Chueh, K., Conley, C. and Smoller, J., 1977, Positively invariant regions for systems of nonlinear diffusion equations, Indiana University Mathematics Journal, 26, 373-392.

[8] Gantmacher, F.R., 1959, Applications of the Theory of Matrices (New York, NY: Interscience Publishers).

[9] Grindrod, P., 1991, Patterns and Waves (Oxford: Clarendon Press).

[10] Henry, D., 1981, Geometric Theory of Semilinear Parabolic Equations (Berlin: Springer-Verlag).

[11] Hill, C.A., 1984, Bronchioalveolar carcinoma: A review, Radiology, 150, 15-20.

[12] Kakinuma, R., Ohmatsu, H., Kaneko, M., Kusumoto, M., Yoshida, J., Nagai, K., Nishiwaki, Y., Kobayashi, T., Tsuchiya, R., Nishiyama, H., Matsui, E., Eguchi, K. and Moriyama, N., 2004, Progression of focal pure groundglass opacity detected by low-dose helical computed tomography screening for lung cancer, Journal of Computer Assisted Tomography, 28, 17-23.

[13] Kerr, K.M., 2001, Pulmonary preinvasive neoplasia, Journal of Clinical Pathology, 54, 257-271.

[14] Kerr, K.M., MacKenzie, S.J., Ramasami, S., Murray, G.I., Fyfe, N., Chapman, A.D., Nicolson, M.C. and King, G., 2004, Expression of Fhit, cell adhesion molecules and matrix metalloproteinases in atypical adenomatous hyperplasia and pulmonary adenocarcinoma, Journal of Pathology, 203, 638-644.

[15] Kimmel, M. and Traganos, F., 1986, Estimation and prediction of cell cycle specific effects of anticancer drugs, Mathematical Biosciences, 80, 187-208.

[16] Marciniak-Czochra, A., 2004, Developmental models with cell surface receptor densities defining morphological position, SFB Preprint. University of Heidelberg.

[17] Marciniak-Czochra, A. and Kimmel, M., 2006, Reaction-diffusion approach to modelling of the spread of early tumors along linear or tubular structures, Journal of Theoretical Biology, in press.

[18] Marciniak-Czochra, A. and Ptashnyk, M., 2005, Derivation of a macroscopic receptor-based model using homogenisation techniques. Submitted.

[19] Moolgavkar, S.H., 1988, Biologically motivated two-stage model for cancer risk assessment, Toxicology Letters, 43, 139-150.

[20] Murray, J.D., 2003, Mathematical Biology (Berlin: Springer-Verlag).

[21] Niho, S., Yokose, T., Suzuki, K., et al., 1999, Monoclonality of atypical adenomatous hyperplasia of the lung, American Journal of Pathology, 154, 249-254.

[22] Smoller, J., 1994, Shock-Waves and Reaction-diffusion Equations (New York, NY: Springer-Verlag).

[23] Travis, W.T., Garg, K., Franklin, W.A., Wistuba, I.I., Sabloff, B., Noguchi, M., Kakinuma, R., Zakowski, M., Ginsberg, M., Padera, R., Jacobson, F., Johnson, B.E., Hirsch, F., Brambilla, E., Flieder, D.B., Geisinger, K.R., Thunnisen, F., Kerr, K., Yankelevitz, D., Franks, T.J., Galvin, J.R., Henderson, D.W., Nicholson, A.G., Hasleton, P.S., Roggli, V., Tsao, M.-S., Cappuzzo, F. and Vazqueza, M., 2005, Evolving concepts in the pathology and computed tomography imaging of lung adenocarcinoma and bronchioloalveolar carcinoma, Journal of Clinical Oncology, 23, 3279-3287.

\section{Appendix}

In this Appendix, we present linear stability analysis of system (10), which leads to the formulation of the necessary and sufficient conditions for diffusion driven instabilities in the model.

Let $A$ be the Jacobian matrix computed at the spatially homogeneous steady state. Linear stability of the homogeneous steady state to spatially heterogeneous perturbations is determined by the sign of $\operatorname{Re} \lambda\left(\mu_{n}^{2}\right)$, where $\lambda\left(\mu_{n}^{2}\right)$ belongs to the spectrum of $\tilde{A}\left(\mu_{n}^{2}\right)$ and

$$
\tilde{A}\left(\mu_{n}^{2}\right)=A-\delta D,
$$


where $D$ is the matrix of diffusion coefficients,

$$
D=\left(\begin{array}{ccc}
0 & 0 & 0 \\
0 & 0 & 0 \\
0 & 0 & 1
\end{array}\right)
$$

and $\delta=\mu_{n}^{2} / \gamma$ involves the wavenumber obtained from the Laplacian's eigenproblem:

$$
\begin{gathered}
\Delta_{x} \phi_{m}=-\mu_{m}^{2} \phi_{m} \text { in } \Omega, \\
\partial_{n} \phi_{m}=0 .
\end{gathered}
$$

When the perturbing mode with an appropriate wavenumber is unstable, it can grow. From the linear stability analysis we obtain the dispersion relation $\lambda=\lambda\left(\mu_{m}^{2}\right)$ as a solution of the characteristic polynomial,

$$
|\tilde{A}-\lambda I|=0 .
$$

where $|A|$ denotes the determinant of matrix $A$. Note that $\gamma$ occurs in the system in such way that we may consider $\lambda=\lambda\left(\mu_{m}^{2} / \gamma\right)$ with $\gamma$ appearing nowhere explicitly. Function $\lambda\left(\mu_{m}^{2} / \gamma\right)$ as a function of $\gamma$ for different $\mu_{m}^{2}$ is simply scaled along the $\gamma$-axis.

Conditions for linearised stability of the kinetics system of three equations around the spatially homogeneous steady state can be derived using the Routh-Hurwitz theory [8]. Let us recall that for the system of three linearised ODEs the characteristic polynomial has the form

$$
\chi_{A}(\lambda)=\lambda^{3}-t r A \lambda^{2}+\sum_{i<j}\left|A_{i j}\right| \lambda-|A|=0
$$

$A_{i_{1} i_{2}}$ denotes the submatrix of $A$ consisting of the $i_{1}$-th and $i_{2}$-th column and $i_{1}$-th and $i_{2}$-th row and $\left|A_{i_{1} i_{2}}\right|=a_{i_{1} i_{1}} a_{i_{2} i_{2}}-a_{i_{1} i_{2}} a_{i_{2} i_{1}}$.

The Hurwitz matrix has the form,

$$
H=\left(\begin{array}{lll}
-\sigma_{1} & -\sigma_{3} & 0 \\
1 & \sigma_{2} & 0 \\
0 & -\sigma_{1} & -\sigma_{3}
\end{array}\right)=\left(\begin{array}{lll}
-t r A & -|A| & 0 \\
1 & \sum_{i<j}\left|A_{i j}\right| & 0 \\
0 & -t r A & -|A|
\end{array}\right) .
$$

Let $\Delta_{i}$ be the determinants of successive principal minors of matrix $H$,

$$
\begin{aligned}
& \Delta_{1}=-\operatorname{tr} A=-\sigma_{1}, \\
& \Delta_{2}=-\operatorname{tr} A\left(\sum_{i<j}\left|A_{i j}\right|\right)+|A|=-\sigma_{1} \sigma_{2}+\sigma_{3}, \\
& \Delta_{3}=-\Delta_{2}|A|=-\left(-\sigma_{1} \sigma_{2}+\sigma_{3}\right) \sigma_{3} .
\end{aligned}
$$


From Routh-Hurwitz Theorem [8]: The number $k$ of roots of the real polynomial which lie in the right half-plane is given by the formula,

$$
k=V\left(1, \Delta_{1}\right)+V\left(\Delta_{1}, \Delta_{3}\right)+V\left(1, \Delta_{2}\right),
$$

where $V$ is the number of changes of sign of adjacent numbers in a sequence.

For the ODE system, $k=0$, i.e. the equilibrium is asymptotically stable, if and only if all $\Delta_{i}>0$. For the reaction-diffusion system, the characteristic polynomial has the following form,

$$
\chi_{\tilde{A}}(\lambda)=\lambda^{3}-\operatorname{tr} \tilde{A} \lambda^{2}+\sum_{i<j}\left|\tilde{A}_{i j}\right| \lambda-|\tilde{A}|=0,
$$

and determinants of the principal minors of the Hurwitz matrix read,

$$
\begin{aligned}
& \tilde{\Delta}_{1}=-\operatorname{tr} A+\frac{1}{\gamma} \mu_{m}^{2}=\Delta_{1}+\frac{1}{\gamma} \mu_{m}^{2}, \\
& \tilde{\Delta}_{2}=\left(-t r A+\frac{1}{\gamma} \mu_{m}^{2}\right)\left(\sum_{i<j}\left|A_{i j}\right|-\operatorname{tr} A_{12} \frac{1}{\gamma} \mu_{m}^{2}\right)+\left(|A|-\left|A_{12}\right| \frac{1}{\gamma} \mu_{m}^{2}\right) \\
&=-\operatorname{tr} A_{12}\left(\frac{1}{\gamma}\right)^{2} \mu_{m}^{4}+\left(\left|A_{23}\right|+\left|A_{13}\right|+\operatorname{tr} A_{12} \operatorname{tr} A\right) \frac{1}{\gamma} \mu_{m}^{2}+\Delta_{2}, \\
& \tilde{\Delta}_{3}=-\tilde{\Delta}_{2}|\tilde{A}|=\tilde{\Delta}_{2}\left(-|A|+\left|A_{12}\right| \frac{1}{\gamma} \mu_{m}^{2}\right) .
\end{aligned}
$$

For a diffusion-driven instability we first require that $\operatorname{Re} \lambda\left(\mu_{m}^{2}\right)<0$ for $\mu_{m}^{2}=0$, that is, the uniform steady state is stable in the absence of diffusion. This leads to the conditions,

$$
\begin{gathered}
\operatorname{Re} \lambda\left(\mu_{m}^{2}=0\right)<0 \equiv-\operatorname{tr} \quad A>0 \\
\wedge-\operatorname{tr} A\left(\sum_{i<j}\left|A_{i j}\right|\right)+|A|>0 \\
\wedge-|A|>0 .
\end{gathered}
$$

Second, we require that there exists a positive $\mu_{m}^{2}$, for which $\lambda\left(\mu_{m}^{2}\right)$ has a positive real part. Using the Routh-Hurwitz conditions we obtain,

$$
\begin{gathered}
\operatorname{Re} \lambda\left(\mu_{m}^{2}\right)>0 \equiv \tilde{\Delta}_{1}<0, \\
\vee \tilde{\Delta}_{2}<0, \\
\vee \tilde{\Delta}_{3}<0 .
\end{gathered}
$$


Adding diffusive terms to the trace can only decrease the trace and therefore increase $\tilde{\Delta}_{1}$. Therefore, $\tilde{\Delta}_{1}$ is always positive if $\Delta_{1}$ is positive. We analyze the above conditions (34)-(39) and obtain that a steady state which is stable in the absence of diffusion can be destabilised due to the diffusion if and only if $\left|A_{12}\right|<0$.

Lemma 8.1. For the system (10) a steady state which is stable in the absence of diffusion (conditions (34)-(36) are fulfilled), is destabilised when diffusion is introduced if and only if $\left|A_{12}\right|<0$.

Proof. Sufficiency: If $\left|A_{12}\right|<0$ then there exists $\left(\mu_{m}^{2} / \gamma\right)>0$ such that

$$
-|\tilde{A}|=-|A|+\left|A_{12}\right| \frac{1}{\gamma} \mu_{m}^{2}<0 .
$$

If $\tilde{\Delta}_{2}>0$ then $\tilde{\Delta}_{3}<0$ and if $\tilde{\Delta}_{2}<0$ then $\tilde{\Delta}_{3}>0$, i.e. in both cases $k=1$.

Necessity: Assume that $\left|A_{12}\right|>0$. Then $a_{11}<0$ and also $\operatorname{tr} A<0$ and $\operatorname{tr} A_{12}<0$. We can show that $\tilde{\Delta}_{2}>0$. Indeed, assume that $\tilde{\Delta}_{2}<0$ in any $(1 / \gamma) \mu_{m}^{2}$. Then,

$$
\tilde{\Delta}_{2}=-t r A_{12}\left(\frac{1}{\gamma}\right)^{2} \mu_{m}^{4}+\left(\left|A_{23}\right|+\left|A_{13}\right|+\operatorname{tr} A_{12} \operatorname{tr} A\right) \frac{1}{\gamma} \mu_{m}^{2}+\Delta_{2}<0
$$

only if

$$
\left(\left|A_{23}\right|+\left|A_{13}\right|+t r A_{12} t r A\right)<0 .
$$

From this condition we obtain

$$
a_{23} a_{32}>\left(a_{11}+a_{22}\right)^{2}+2\left(a_{11}+a_{22}\right) a_{33}-a_{13} a_{31} .
$$

On the other hand if $\Delta_{2}>0$, then $\sum_{i<j}\left|A_{i j}\right|>0$. This leads to the following condition,

$$
a_{23} a_{32}<a_{11} a_{22}+a_{11} a_{33}+a_{22} a_{33}-a_{13} a_{31}-a_{12} a_{21} .
$$

Combining the two above inequalities we obtain

$$
a_{11}^{2}+a_{22}^{2}+a_{11} a_{22}+a_{11} a_{33}+a_{22} a_{33}<-a_{12} a_{21},
$$

which cannot be verified considering the signs of $a_{i j}$ and that $a_{11}<0$.

If $\left|A_{12}\right|>0$ then also $\tilde{\Delta}_{3}>0$ for every $\mu_{\mathrm{m}}$. Therefore, for $\left|A_{12}\right|>0, \tilde{\Delta}_{i}>0, i=1,2,3$ and the destabilisation of the steady state is impossible. This concludes the proof. 


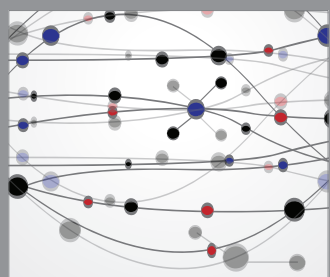

The Scientific World Journal


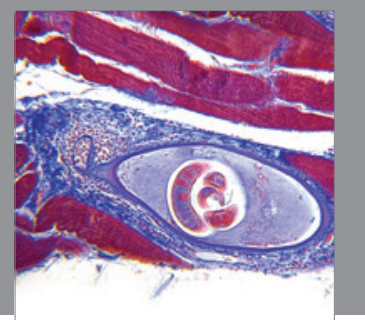

Gastroenterology

Research and Practice


\section{Hindawi}

Submit your manuscripts at

http://www.hindawi.com
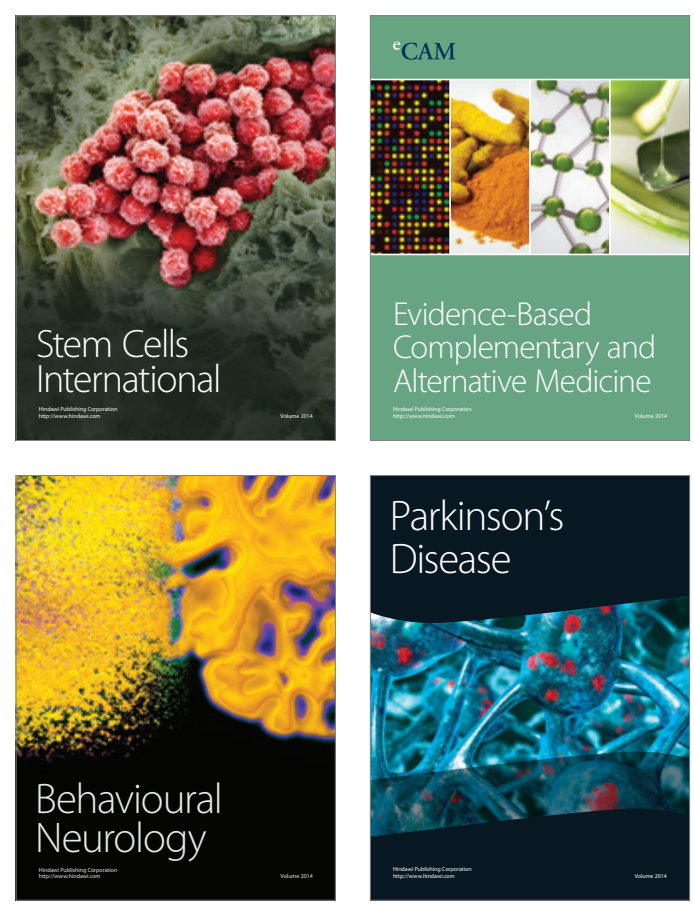

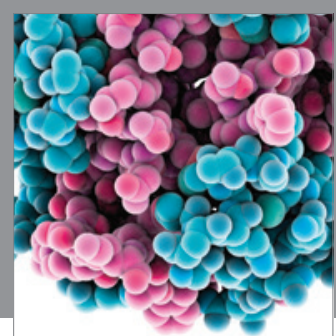

Journal of
Diabetes Research

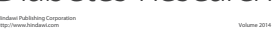

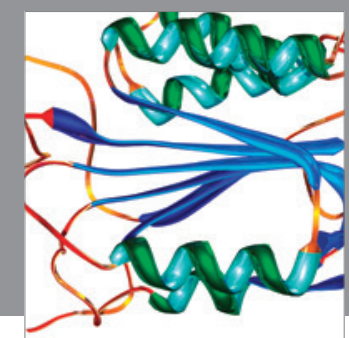

Disease Markers
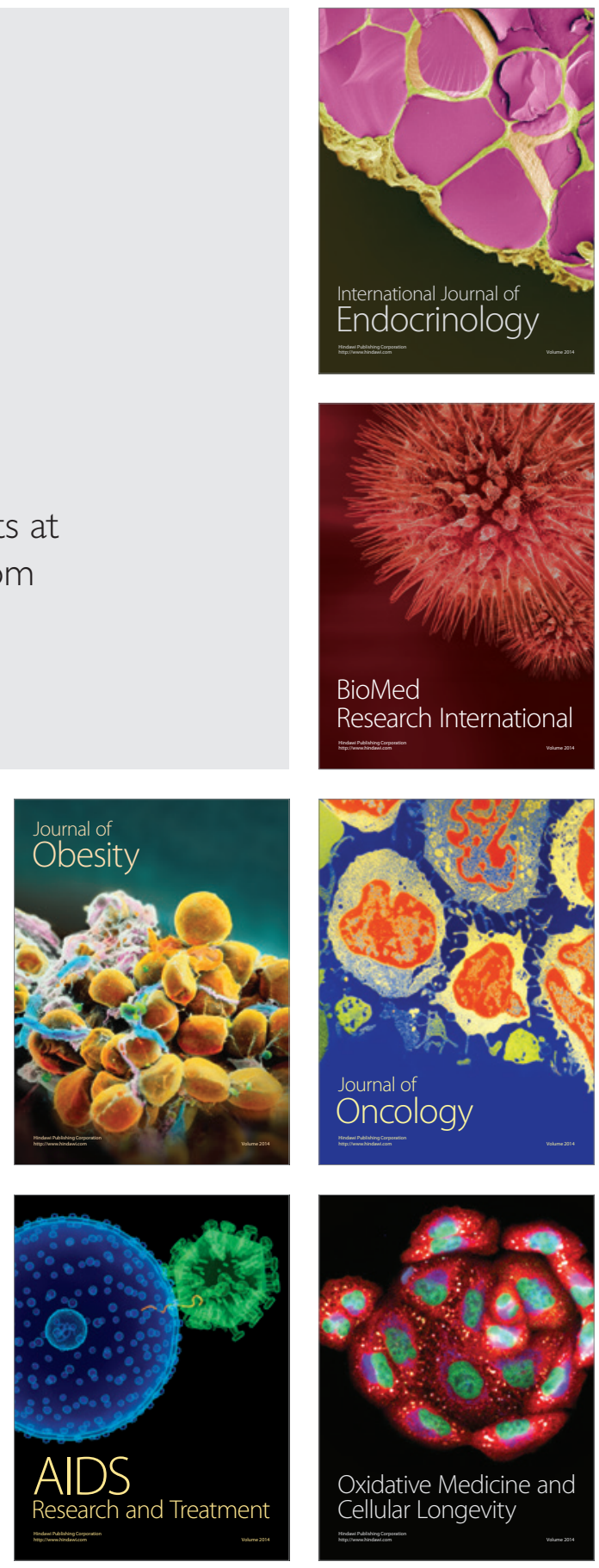